\title{
Desempenho e rendimento de carcaça de frangos de corte alimentados com pectina na ração
}

\author{
[Performance and carcass yield of broiler chickens fed with pectin in the ration] \\ V.K. Silva, V.S. Morita, I.C. Boleli*
}

Universidade Estadual Paulista - UNESP/Jaboticabal, SP

\begin{abstract}
RESUMO
Avaliou-se o efeito da ingestão contínua de pectina em 720 frangos de corte sobre o desempenho e rendimento de carcaça e o consumo de água. O delineamento foi inteiramente ao acaso, em esquema de parcela subdividida, com níveis de pectina $0,1,3$ e 5\%, e idade primeira, segunda, terceira, quarta, quinta e sexta semanas, com seis repetições por tratamento. A viabilidade criatória não foi influenciada pela pectina na ração. O peso vivo, o consumo de ração e o ganho de peso diminuíram, e a conversão alimentar aumentou com a ingestão de pectina aos 35 e 42 dias. Não houve efeito da pectina sobre o consumo de ração (CR) e o consumo de água (CAg) na terceira semana. Na sexta semana, o CAg e a relação CAg:CR aumentaram com a ingestão de pectina. Aos $35 \mathrm{e} 42$ dias, todos os parâmetros avaliados para rendimento de carcaça e corte diminuíram com o aumento da ingestão da fibra, sendo que apenas o rendimento de coxa+sobrecoxa não sofreu influência aos 42 dias. Os dados mostram que a ingestão contínua de até $1 \%$ de pectina na ração mantém o desempenho máximo das aves e que o consumo acima desse nível prejudica os parâmetros zootécnicos e aumenta o consumo de água na fase de maior crescimento.
\end{abstract}

Palavras-chave: frango, aditivo, carcaça, consumo de água, fibra solúvel

\begin{abstract}
The effect of the continuous intake of pectin was evaluated for 720 broilers regarding performance, carcass yield and water consumption. The design was completely randomized in a split plot design, with pectin levels of $0,1,3$ and $5 \%$ and age $1^{\text {st }}, 2^{\text {nd }}, 3^{\text {rd }}, 4^{\text {th }}, 5^{\text {th }}$ and $6^{\text {th }}$ week, with six replicates. The viability was not influenced by dietary pectin. Body weight, feed intake and weight gain decreased and feed intake increased with pectin at 35 and 42 days. No effect of pectin on feed intake (FI) and water consumption $(W)$ in the third week. On the sixth week regarding W and FI: W increased with the intake of pectin. At 35 and 42 days all parameters for carcass and cut decreased with increasing intake of fiber, and only the thigh + drumstick yield was not affected at 42 days. The data show that the continuous intake of up to $1 \%$ pectin in the diet maintains the best performance in birds and consumption above that level affects the performance parameters and increases the consumption of water in the higher growth phase.
\end{abstract}

Keywords: broiler, addictive, carcass, soluble fiber, water intake

\section{INTRODUÇÃO}

Atualmente a avicultura brasileira procura utilizar-se de manejo adequado para atingir a expressão máxima do potencial genético produtivo das linhagens de corte, o que envolve, necessariamente, o desenvolvimento e o aprimoramento do manejo nutricional, da sanidade e do bem-estar animal.

Nos últimos anos, o uso de aditivos que possuam atividade benéfica aos animais também tem sido pesquisado. Dentre esses aditivos, a utilização de fibras solúveis, principalmente a pectina, na

Recebido em 25 de março de 2011

Aceito em 13 de abril de 2012

*Autor para correspondência (corresponding author)

E-mail: icboleli@fcav.unesp.br 
alimentação de animais tem merecido destaque por alterar o metabolismo lipídico (Delzenne et al., 2002; Carr et al., 2003; Takahashi e Sakata, 2005), o funcionamento cecal (Juśkiewicz et al., 2006), a microbiota intestinal e a resposta inflamatória intestinal (Teirlynck et al., 2009; Saki et al., 2010), bem como prevenir a formação de lesões intestinais (Satoh et al., 2010). Os efeitos da ingestão de fibra solúvel têm sido relacionados com a alteração da viscosidade intestinal que ocorre com sua ingestão e que resulta de sua propriedade de absorver água e consequente geleificação (Smits e Annison, 1996; Fietz e Salgado, 1999; Rosa e Uttpatel, 2007). No entanto, a alteração na viscosidade intestinal pode dificultar a digestão e a absorção dos nutrientes (Van de Klis et al., 1993, Smits et al., 1997), além de promover redução na ingestão de ração (Serena et al., 2007). Esses efeitos negativos podem reduzir o desenvolvimento e o desempenho das aves, principalmente nas primeiras semanas de vida, quando o máximo desenvolvimento do sistema digestório é alcançado.

Do ponto de vista nutricional, o bom desempenho das aves depende da ingestão adequada de energia e de compostos, como água, sais minerais, lipídios, carboidratos, vitaminas e proteínas, pelo organismo (Boaro, 2009). Para maximização da expressão do potencial de crescimento animal, o desejável é que os benefícios promovidos pelo uso de fibras solúveis na dieta venham acompanhados da manutenção e/ou melhora do desempenho atual das linhagens utilizadas.

Assim, o presente estudo teve o objetivo de avaliar os efeitos da adição de fibra solúvel pectina sobre o desenvolvimento, o desempenho, o rendimento de carcaça e de cortes nobres e a ingestão de água por frangos de corte.

\section{MATERIAL E MÉTODOS}

O experimento foi realizado após aprovação do protocolo experimental pela Comissão de Ética no Uso de Animais - CEUA, da mesma instituição (protocolo número 21.122/10).

Setecentos e vinte pintos machos $\left(\mathrm{Cobb}^{\circledR}\right)$, com um dia de idade, obtidos de incubatório comercial, foram pesados individualmente e divididos em quatro tratamentos $0,1,3$ e $5 \%$ de pectina na ração, sendo 180 aves por tratamento. As aves foram distribuídas homogeneamente pelo peso médio corporal $(45 \pm 0,02 \mathrm{~g})$, em boxes (2,50x1,50m), em galpão de alvenaria, sendo seis boxes por tratamento (30 aves/box). Os boxes foram forrados com casca de arroz e equipados com bebedouros de alumínio tipo copo de pressão, comedouro tubular infantil e fonte de aquecimento constituída de lâmpadas incandescentes de 200 watts. Após o sétimo dia de idade, foram utilizados bebedouros pendulares e comedouros tubulares com capacidade de $25 \mathrm{~kg}$. A partir do $14^{\circ}$ dia de idade, as aves foram submetidas ao regime diário de 23 h de luz e uma hora de escuro.

As aves foram vacinadas contra as doenças de Marek e bouba aviária, no primeiro dia de vida, ainda no incubatório, de Gumboro (cepa intermediária Lukert) e de Newcastle (estirpe La Sota) no oitavo dia, e de Gumboro (cepa forte Austrália V-877) aos 18 dias.

A temperatura e a umidade relativa no interior do galpão foram registradas durante todo o período experimental, utilizando-se dois termohigrômetros digitais localizados em pontos equidistantes do galpão. As temperaturas foram mensuradas duas vezes ao dia, registrando-se seus valores máximo e mínimo, sendo as médias de $\quad 31,3 \pm 2,7^{\circ} \mathrm{C}, \quad 29,3 \pm 2,4^{\circ} \mathrm{C}, \quad 27,3 \pm 1,5^{\circ} \mathrm{C}$, $26,4 \pm 1,3^{\circ} \mathrm{C}, \quad 23,0 \pm 1,3^{\circ} \mathrm{C}, \quad 23,0 \pm 1,1^{\circ} \mathrm{C}$ e de $40,8 \pm 3,5 \%, 50,0 \pm 4,0 \%, 58,0 \pm 3,2 \%, 54,9 \pm 3,6 \%$, $53,0 \pm 3,3 \%, 51,8 \pm 2,8 \%$, da primeira à sexta semanas do experimento, respectivamente.

As rações foram à base de milho e farelo de soja (Tab. 1), reajustadas de acordo com as recomendações de Rostagno et al. (2005), para as fases inicial (um a 21 dias) e de crescimento (22 a 42 dias) dos frangos. As aves receberam água e ração à vontade durante todo o período experimental.

Ganho de peso (GP), consumo de ração (CR), conversão alimentar $(\mathrm{CA}=\mathrm{GP} / \mathrm{CR})$ e viabilidade criatória (VC) foram avaliados semanalmente e nos períodos de um a 35 e de um a 42 dias de idade.

O consumo de água (CAg) foi avaliado de 14 a 21 e de 35 a 42 dias de idade. Para isso, um galão com capacidade de $5 \mathrm{~L}$ foi acoplado a cada bebedouro. Os equipamentos (galão + 
mangueiras + bombas + bebedouro pendular) foram pesados inicialmente sem água e identificados. Após esse procedimento, os galões foram enchidos com água e todos os equipamentos foram pesados novamente, anotando-se individualmente seus pesos, para a obtenção do peso da água contida em cada um deles. Diariamente, nos sete dias seguintes, os equipamentos contendo a água não ingerida foram pesados e, pela diferença de peso, calculou-se a quantidade de água ingerida em cada dia. Para controle da perda de água por evaporação, um equipamento, contendo água e previamente pesado, foi colocado no galpão e utilizado para o cálculo da perda evaporativa diária de água. Esse valor foi utilizado nos cálculos do consumo real de água. O consumo de água foi calculado como o consumo médio diário por ave, dividindo-se o consumo diário de água no box pelo número total de aves. A relação entre o consumo médio diário de água e de ração também foi avaliada para os dois períodos.

Tabela 1. Composição percentual e nutricional das dietas usadas para frangos de corte

\begin{tabular}{|c|c|c|c|c|c|c|c|c|}
\hline \multirow{2}{*}{ Ingrediente } & \multicolumn{4}{|c|}{ Inicial } & \multicolumn{4}{|c|}{ Crescimento } \\
\hline & $0 \%$ & $1 \%$ & $3 \%$ & $5 \%$ & $0 \%$ & $1 \%$ & $3 \%$ & $5 \%$ \\
\hline Milho & 47,50 & 47,50 & 47,50 & 47,50 & 52,50 & 52,50 & 52,50 & 52,50 \\
\hline Farelo de soja & 36,92 & 36,92 & 36,92 & 36,92 & 30,95 & 30,95 & 30,95 & 30,95 \\
\hline Óleo de soja & 5,75 & 5,75 & 5,75 & 5,75 & 7,40 & 7,40 & 7,40 & 7,40 \\
\hline Fosfato bicálcico & 1,90 & 1,90 & 1,90 & 1,90 & 1,64 & 1,64 & 1,64 & 1,64 \\
\hline Calcário calcítico & 0,82 & 0,82 & 0,82 & 0,82 & 0,75 & 0,75 & 0,75 & 0,75 \\
\hline Sal & 0,47 & 0,47 & 0,47 & 0,47 & 0,43 & 0,43 & 0,43 & 0,43 \\
\hline $\begin{array}{l}\text { L-Lisina HCL } \\
(78 \%)\end{array}$ & 0,61 & 0,61 & 0,61 & 0,61 & 0,30 & 0,30 & 0,30 & 0,30 \\
\hline $\begin{array}{l}\text { DL-Metionina } \\
(99 \%)\end{array}$ & 0,34 & 0,34 & 0,34 & 0,34 & 0,40 & 0,40 & 0,40 & 0,40 \\
\hline L-Treonina & 0,14 & 0,14 & 0,14 & 0,14 & 0,08 & 0,08 & 0,08 & 0,08 \\
\hline BHT & 0,05 & 0,05 & 0,05 & 0,05 & 0,05 & 0,05 & 0,05 & 0,05 \\
\hline $\begin{array}{l}\text { Supl. vitamínico e } \\
\text { mineral }\end{array}$ & $0,50^{1}$ & $0,50^{1}$ & $0,50^{1}$ & $0,50^{1}$ & $0,50^{2}$ & $0,50^{2}$ & $0,50^{2}$ & $0,50^{2}$ \\
\hline Inerte $^{3}$ & 5,00 & 4,00 & 2,00 & 0,00 & 5,00 & 4,00 & 2,00 & 0,00 \\
\hline Pectina $^{4}$ & 0,00 & 1,00 & 3,00 & 5,00 & 0,00 & 1,00 & 3,00 & 5,00 \\
\hline TOTAL & 100,00 & 100,00 & 100,00 & 100,00 & 100,00 & 100,00 & 100,00 & 100,00 \\
\hline & \multicolumn{8}{|c|}{ Composição calculada } \\
\hline $\begin{array}{l}\text { Energia } \\
\text { metabolizável } \\
(\mathrm{kcal} / \mathrm{kg})\end{array}$ & 3.000 & 3.000 & 3.000 & 3.000 & 3.175 & 3.175 & 3.175 & 3.175 \\
\hline Proteína bruta (\%) & 21,62 & 21,62 & 21,62 & 21,62 & 19,02 & 19,02 & 19,02 & 19,02 \\
\hline $\mathrm{Ca}(\%)$ & 0,92 & 0,92 & 0,921 & 0,92 & 0,81 & 0,81 & 0,81 & 0,81 \\
\hline $\operatorname{Pd}(\%)$ & 0,46 & 0,46 & 0,46 & 0,46 & 0,40 & 0,40 & 0,40 & 0,40 \\
\hline $\mathrm{Na}(\%)$ & 0,22 & 0,22 & 0,22 & 0,22 & 0,20 & 0,20 & 0,20 & 0,20 \\
\hline Lis dig (\%) & 1,50 & 1,50 & 1,50 & 1,50 & 1,13 & 1,13 & 1,13 & 1,13 \\
\hline Met dig (\%) & 0,63 & 0,63 & 0,63 & 0,63 & 0,66 & 0,66 & 0,66 & 0,66 \\
\hline Met+Cis $\operatorname{dig}(\%)$ & 0,91 & 0,91 & 0,91 & 0,91 & 0,91 & 0,91 & 0,91 & 0,91 \\
\hline Thr dig $(\%)$ & 0,83 & 0,83 & 0,83 & 0,83 & 0,70 & 0,70 & 0,70 & 0,70 \\
\hline
\end{tabular}

${ }^{1}$ Suplemento mineral + vitamínico. Cada quilograma do produto contém: Se 54,6mg, Cu 25.000mg, pantotenato de Ca $1.900 \mathrm{mg}$, Mn $15.252 \mathrm{mg}$, I 260mg, Zn $18.250 \mathrm{mg}$, ácido nicotínico $6.930 \mathrm{mg}$, biotina $32 \mathrm{mg}$, colina $120 \mathrm{~g}$, Vit. A 1.400 .000 UI, Vit. B1 356mg, Vit. B12 2.000mcg, Vit. B2 $1.920 \mathrm{mg}$, Vit. B6 693mg, Vit. D3 600.000, Vit. E $5.000 \mathrm{mg}$, Vit. K $196 \mathrm{mg}$, antioxidante $100 \mathrm{mg}$, anticoccidiano $25.000 \mathrm{mg}$ e promotor de crescimento (bacitracina de zinco) $10.000 \mathrm{mg}$.

${ }^{2}$ Suplemento mineral + vitamínico. Cada quilograma do produto contém: Se 54,6mg, Cu 25.000mg, pantotenato de Ca 1.900mg, niacina 69,30mg, Mn 15.252mg, I 260mg, Zn 18.250mg, biotina 32mg, DL-metionina 270g, colina 120 g, Vit. A 1.400 .000 UI, Vit. B1 356mg, Vit. B12 2.000mcg, Vit. B2 1.920mg, Vit. B6 693mg, Vit. D 600.000, Vit. E $5.000 \mathrm{mg}$, Vit. K $196,5 \mathrm{mg}$, antioxidante $100 \mathrm{mg}$, coccidiostático $22.000 \mathrm{mg}$ e promotor de crescimento (bacitracina de zinco) $10.000 \mathrm{mg}$.

${ }^{3}$ Inerte: caulin.

${ }^{4}$ Pectina de alto grau de esterificação, alto grau de gelificação, alto teor de carboidratos solúveis (85\%), não amidada, extraída de polpa cítrica (pectina GENUß tipo B rapid set-Z, CP Kelco, Limeira, Brasil). 
O rendimento de carcaça e de cortes foi avaliado aos 35 e 42 dias de idade. Para isso, duas aves por repetição foram submetidas a jejum de oito horas, pesadas e, em seguida, submetidas aos procedimentos usuais de atordoamento, sangria, depenagem e evisceração. $O$ rendimento de carcaça foi calculado em relação ao peso vivo no momento do abate, e os rendimentos de peito e de coxa+sobrecoxa em relação ao peso da carcaça sem os pés, o pescoço, a cabeça, as vísceras e a gordura abdominal.

Os dados foram verificados quanto à presença de outliers e testaram-se as pressuposições de normalidade dos erros estudentizados (teste de Cramer-von-Misses) e de homogeneidade de variâncias (teste de Levene). Depois de constatada a não violação dessas pressuposições, os dados foram submetidos à análise de variância e posterior análise de regressão (linear ou quadrática) por meio do procedimento GLM do programa SAS $^{\circledR} \quad$ (Statistical..., 2002), considerando-se $\alpha=0,05$.

\section{RESULTADOS}

Os efeitos da inclusão de pectina e da idade das aves (dada em semanas) sobre o ganho de peso
(GP), o consumo de ração (CR), a conversão alimentar (CA) e a viabilidade criatória (VC) estão apresentados na Tab. 2. A variável VC foi influenciada apenas pela idade, porém a regressão não foi $(\mathrm{P}>0,05)$. Houve interação de porcentagem de pectina na ração versus a idade para GP, CR e CA $(\mathrm{P} \leq 0,05)$, sendo os desdobramentos da interação apresentados na Tab. 3, e as equações de regressão obtidas para as três variáveis de desempenho na Tab. 4.

O CR aumentou de forma linear $(\mathrm{P} \leq 0,05)$ em função da idade em todas as porcentagens de pectina utilizadas (Tab. 3). Além disso, ocorreu efeito $(\mathrm{P} \leq 0,05)$ das porcentagens de pectina sobre essa variável na terceira, quarta e quinta semanas de idade. O CR em função da adição de pectina foi descrito por efeito quadrático na terceira e quinta semanas e por efeito linear na quarta semana. Na terceira semana, foi estimado maior CR com a inclusão de $2,185 \%$ de pectina. $\mathrm{Na}$ quarta semana, o CR aumentou com o aumento no nível de pectina, sendo maior para as aves que receberam 5\%. Na quinta semana, estimou-se o maior $\mathrm{CR}$ com a inclusão de $1,861 \%$ de pectina.

Tabela 2. Consumo de ração, ganho de peso, conversão alimentar e viabilidade de frangos de corte, de acordo com a porcentagem de pectina e a idade das aves

\begin{tabular}{ccccc}
\hline $\begin{array}{c}\text { Pectina } \\
(\%)\end{array}$ & Consumo de ração $(\mathrm{g})$ & $\begin{array}{c}\text { Ganho de peso } \\
(\mathrm{g})\end{array}$ & $\begin{array}{c}\text { Conversão } \\
\text { alimentar* }\end{array}$ & $\begin{array}{c}\text { Viabilidade criatória } \\
(\%)\end{array}$ \\
\hline 0 & 763 & 454 & 1,53 & 98,87 \\
1 & 692 & 460 & 1,44 & 99,69 \\
3 & 745 & 410 & 1,70 & 99,59 \\
5 & 678 & 285 & 2,19 & 99,06 \\
\hline Idade (semanas) & & & & 99,58 \\
1 & 134 & 139 & 0,99 & 99,85 \\
2 & 301 & 204 & 1,52 & 99,42 \\
3 & 553 & 312 & 1,82 & 100,00 \\
4 & 1022 & 530 & 2,05 & 98,01 \\
5 & 1121 & 602 & 2,02 & 98,98 \\
6 & 1182 & 625 & 1,91 & 0,1273 \\
P & $<0,0001$ & $<0,0001$ & $<0,0001$ & $<0,0001$ \\
I & $<0,0001$ & $<0,0001$ & $<0,0001$ & 0,6960 \\
P x I & $<0,0001$ & $<0,0001$ & $<0,0001$ & $n$ \\
\hline Regressão & - & & \\
I & - & 4,63 & 1,20 \\
CV & 9,73 & 8,14 & &
\end{tabular}


Desempenho e rendimento...

Tabela 3. Desdobramentos da interação de porcentagem de pectina na ração versus a idade para ganho de peso, consumo de ração e conversão alimentar de frangos

\begin{tabular}{|c|c|c|c|c|c|c|}
\hline \multirow{2}{*}{$\begin{array}{c}\text { Idade } \\
\text { (semanas) }\end{array}$} & \multicolumn{4}{|c|}{ Pectina $(\%)$} & \multirow{2}{*}{ Linear (L) } & \multirow{2}{*}{$\begin{array}{c}\text { Quadrática } \\
\text { (Q) }\end{array}$} \\
\hline & 0 & 1 & 3 & 5 & & \\
\hline \multicolumn{7}{|c|}{ Consumo de ração $(\mathrm{g})$} \\
\hline 1 & 134 & 136 & 142 & 124 & 0,5179 & 0,2298 \\
\hline 2 & 297 & 328 & 318 & 259 & 0,3723 & 0,1098 \\
\hline 3 & 557 & 594 & 596 & 467 & 0,0576 & $0,0426^{1}$ \\
\hline 4 & 950 & 962 & 1044 & 1135 & $\mathbf{0 , 0 1 0 3}$ & 0,3220 \\
\hline 5 & 1176 & 1196 & 1156 & 969 & 0,0877 & $0,0438^{2}$ \\
\hline 6 & 1462 & 1250 & 1212 & 1114 & 0,0985 & 0,5759 \\
\hline $\mathbf{L}$ & $<0,0001$ & 0,0425 & $\mathbf{0 , 0 0 1 2}$ & 0,0091 & & \\
\hline Q & 0,4478 & 0,3808 & 0,3107 & 0,5008 & & \\
\hline \multicolumn{7}{|c|}{ Ganho de peso (g) } \\
\hline 1 & 145 & 159 & 130 & 123 & 0,1640 & 0,8336 \\
\hline 2 & 223 & 229 & 224 & 139 & 0,1736 & 0,1311 \\
\hline 3 & 358 & 358 & 316 & 215 & 0,0370 & $0,0076^{3}$ \\
\hline 4 & 605 & 608 & 519 & 387 & $\mathbf{0 , 0 3 2 3}$ & 0,1793 \\
\hline 5 & 717 & 722 & 636 & 333 & 0,0806 & 0,0683 \\
\hline 6 & 674 & 680 & 633 & 515 & 0,0532 & $0,0154^{4}$ \\
\hline $\mathrm{L}$ & $\mathbf{0 , 0 0 2 7}$ & 0,0028 & 0,0009 & 0,0046 & & \\
\hline Q & 0,4133 & 0,4721 & 0,5077 & 0,6257 & & \\
\hline \multicolumn{7}{|c|}{ Conversão alimentar* } \\
\hline 1 & 0,94 & 0,87 & 1,12 & 1,02 & 0,3813 & 0,7047 \\
\hline 2 & 1,33 & 1,43 & 1,42 & 1,89 & 0,1169 & 0,3762 \\
\hline 3 & 1,55 & 1,66 & 1,89 & 2,18 & $\mathbf{0 , 0 2 1 0}$ & 0,1450 \\
\hline 4 & 1,57 & 1,70 & 1,90 & 2,93 & 0,0702 & 0,2257 \\
\hline 5 & 1,65 & 1,69 & 1,82 & 2,86 & 0,1092 & 0,1810 \\
\hline 6 & 2,15 & 1,83 & 1,92 & 2,30 & 0,5511 & 0,2005 \\
\hline $\mathrm{L}$ & 0,0043 & 0,0316 & 0,0280 & 0,0129 & & \\
\hline $\mathrm{Q}$ & 0,9952 & 0,0709 & 0,0586 & $\mathbf{0 , 0 2 3 3}^{5}$ & & \\
\hline
\end{tabular}

*Dados comparados utilizando-se variável transformada $=\mathrm{y}^{-0,054}$.

${ }^{1} \mathrm{Y}=-41,5 \mathrm{x}^{2}+180,7 \mathrm{x}+413,23 \mathrm{R}^{2}=0,96$.

${ }^{2} Y=-51,75 x^{2}+192,65 x+1030,8 R^{2}=0,99$.

${ }^{3} R=-9,15 x^{2}+13,82 x+674,46 R^{2}=0,99$.

${ }^{4} R=-7,27 x^{2}+7,79 x+357,82 R^{2}=1$.

${ }^{5} R=-0,15 x^{2}+1,36 x-0,241 \quad R^{2}=0,94$.

O GP aumentou de forma linear significativa $(\mathrm{P} \leq 0,05)$ em função da idade em semanas, independentemente da adição de pectina (Tab. $3)$. Efeitos significativos $(P \leq 0,05)$ dos níveis de pectina sobre o GP foram registrados na terceira, quarta e sexta semanas. O GP em função dos níveis de pectina seguiu efeito quadrático na terceira semana, sendo estimada, para maior GP, a adição de $0,95 \%$ de pectina: na sexta semana, o maior GP foi estimado com o consumo de $0,536 \%$ de pectina. Já na quarta semana, ocorreu efeito linear decrescente em relação ao aumento de pectina na ração, sendo seu menor valor registrado para as aves que receberam ração com $5 \%$ de pectina.
A CA aumentou linearmente ao longo das semanas com a adição de 0,1 e $3 \%$ de pectina e ocorreu efeito quadrático significativo $(\mathrm{P} \leq 0,05)$ para $5 \%$ (Tab. 3). Em relação à idade, observou-se que a CA aumentou linearmente em função das porcentagens de pectina apenas na terceira semana $(\mathrm{P} \leq 0,05)$.

Os resultados para peso vivo (PV), consumo de ração (CR), ganho de peso (GP), conversão alimentar (CA) e viabilidade criatória (VC) nos períodos de um a 35 e de um a 42 dias de idade estão apresentados na Tab. 4. Nos dois períodos analisados, todas essas variáveis, exceto a $\mathrm{VC}$, foram influenciadas significativamente $(\mathrm{P} \leq 0,05)$ pelos níveis de pectina na ração. 
No período de um a 35 dias, o PV, o CR e o GP foram descritos por efeito quadrático significativo $(\mathrm{P} \leq 0,05) \quad$ em função dos níveis de pectina. Os maiores PV e GP foram obtidos com ingestão de pectina estimada em $0,73 \%$ (aproximadamente $2.123 \mathrm{~g}$ e $2.078 \mathrm{~g}$, respectivamente), e os menores foram apresentados pelas aves que ingeriram $5 \%$ da pectina. O maior CR foi estimado em $2,082 \%$ de pectina (em média $3.264 \mathrm{~g}$ ). A CA em função dos níveis de pectina também foi descrita por efeito quadrático (Tab. 4); seus piores valores foram obtidos para as aves que receberam ração com $5 \%$ de pectina, e o melhor foi estimado para o consumo de $0,348 \%$ de pectina.
De um a 42 dias de idade (Tab. 4), o PV e o GP tiveram efeito quadrático significativo $(\mathrm{P} \leq 0,05)$ em função dos níveis de pectina. Seus maiores valores foram obtidos em torno de $1 \%$ de pectina (2.804 e $2.759 \mathrm{~g}$, respectivamente), sendo estimado para maior PV o consumo de $0,849 \%$ de pectina, e seus menores valores foram observados para aves que ingeriram $5 \%$ da fibra na ração. $\mathrm{O} C R$ diminuiu significativa $(\mathrm{P} \leq 0,05)$ e linearmente com o aumento da pectina na ração. A CA foi descrita em função dos níveis por efeito quadrático significativo $(\mathrm{P} \leq 0,05) \quad \mathrm{e}$, diferentemente do observado no período para as demais variáveis, seu pior valor foi estimado com a porcentagem de $1,096 \%$ de pectina na ração.

Tabela 4. Equações de regressão para variáveis de desempenho em frangos de corte alimentados com diferentes porcentagens de inclusão de pectina nos períodos de um a 35 dias e de um a 42 dias de idade

\begin{tabular}{llccc} 
& \multicolumn{1}{c}{ Equação estimada } & $\mathrm{R}^{2}$ & $\mathrm{P}$ & $\mathrm{CV}$ \\
\hline & \multicolumn{1}{c}{$1 \mathrm{a} 35$ dias } & & & \\
Peso vivo & $\mathrm{Y}=-47,62 \mathrm{x}^{2}+69,36 \mathrm{x}+2.096,68$ & 0,9714 & $<0,0001$ & 3,45 \\
Ganho de peso & $\mathrm{Y}=-47,63 \mathrm{x}^{2}+69,39 \mathrm{x}+2.051,31$ & 0,9714 & $<0,0001$ & 3,54 \\
Consumo de ração & $\mathrm{Y}=-41,34 \mathrm{x}^{2}+172,11 \mathrm{x}+3.123,73$ & 0,3779 & 0,0144 & 5,67 \\
Conversão alimentar & $\mathrm{Y}=0,04 \mathrm{x}^{2}+0,03 \mathrm{x}+1,53$ & 0,9564 & 0,0001 & 4,47 \\
Viabilidade & $\mathrm{Y}=97,68$ & - & 0,2781 & 3,30 \\
\hline & \multicolumn{1}{c}{$1 \mathrm{a} 42$ dias } & & & 4,18 \\
Peso vivo & $\mathrm{Y}=-58,48 \mathrm{x}^{2}+99,30 \mathrm{x}+2.745,87$ & 0,9481 & $<0,0001$ & 4,26 \\
Ganho de peso & $\mathrm{Y}=-58,49 \mathrm{x}^{2}+99,33 \mathrm{x}+2.700,5$ & 0,9481 & $<0,0001$ & 4,77 \\
Consumo de ração & $\mathrm{Y}=-95,69 \mathrm{x}+4.624,1$ & 0,4382 & 0,0004 & 4,36 \\
Conversão alimentar & $\mathrm{Y}=0,05 \mathrm{x}^{2}-0,10 \mathrm{x}+1,70$ & 0,9322 & $<0,0001$ & 4,53 \\
Viabilidade & $\mathrm{Y}=96,38$ & - & 0,1034 & 4 \\
\hline
\end{tabular}

De acordo com a Tab. 5, não houve efeito de inclusão de pectina sobre o consumo de água (CAg) na terceira semana (14-21 dias). Contudo, na sexta semana, a CAg aumentou de forma linear $(\mathrm{P} \leq 0,05)$. A relação consumo de água por consumo de ração (CAg:CR) na terceira semana foi descrita por efeito quadrático em função das porcentagens de pectina. A menor relação CAg:CR foi estimada para um consumo de $0,157 \%$ de pectina, e a maior para $5 \%(2,43$ e 3,14 , respectivamente). A relação $\mathrm{CAg:CR}$ na sexta semana aumentou de forma linear $(\mathrm{P} \leq 0,05)$ em função do aumento nos níveis da fibra na ração.

Tabela 5. Equações de regressão para consumo de água (CAg) e relação entre o consumo de água e o consumo de ração (CAg:CR) em frangos de corte na terceira e na sexta semanas de idade

\begin{tabular}{|c|c|c|c|c|}
\hline & Equação estimada & $\mathrm{R}^{2}$ & $\mathrm{P}$ & $\mathrm{CV}(\%)$ \\
\hline \multicolumn{5}{|c|}{ Terceira semana } \\
\hline CAg & $Y=207,12$ & - & 0,1965 & 4,71 \\
\hline CAg : CR & $Y=0,07 x^{2}-0,21 x+2,51$ & 0,6875 & 0,0020 & 8,40 \\
\hline \multicolumn{5}{|c|}{ Sexta semana } \\
\hline CAg & $Y=17,94 x+518,96$ & 0,3043 & 0,0052 & 9,30 \\
\hline CAg : CR & $Y=0,24 x+2,62$ & 0,6950 & $<0,0001$ & 7,78 \\
\hline
\end{tabular}


Os dados sobre o efeito da inclusão de pectina na ração sobre peso de carcaça (PC), rendimento de carcaça (RC), peso de peito (PP), rendimento de peito $(\mathrm{RP})$, peso de coxa+sobrecoxa $(\mathrm{PC}+\mathrm{S})$ e rendimento de coxa+sobrecoxa $(\mathrm{RC}+\mathrm{S})$ de frangos de corte aos 35 e 42 dias de idade são apresentados na Tab. 6 .

Tabela 6. Equações de regressão para rendimento de carcaça de frangos de corte alimentados com diferentes porcentagens de pectina aos 35 e aos 42 dias de idade

\begin{tabular}{lllll}
\hline & \multicolumn{1}{c}{ Equação estimada } & \multicolumn{1}{c}{$\mathrm{R}^{2}$} & $\mathrm{P}$ & $\mathrm{CV}$ \\
\hline & \multicolumn{1}{c}{35 dias } & & & \\
Peso de carcaça & $\mathrm{Y}=-23,28 \mathrm{x}^{2}-14,69 \mathrm{x}+1.499,50$ & 0,8486 & $<0,0001$ & 9,20 \\
Rendimento de carcaça & $\mathrm{Y}=-1,21 \mathrm{x}+73,80$ & 0,8182 & $<0,0001$ & 1,60 \\
Peso de peito & $\mathrm{Y}=-11,54 \mathrm{x}^{2}+11,61 \mathrm{x}+515,39$ & 0,8630 & $<0,0001$ & 8,36 \\
Rendimento de peito & $\mathrm{Y}=-0,25 \mathrm{x}^{2}+0,80 \mathrm{x}+25,21$ & 0,4617 & 0,0025 & 5,15 \\
Peso de coxa+sobrecoxa & $\mathrm{Y}=-5,96 \mathrm{x}^{2}-6,89 \mathrm{x}+427,35$ & 0,8201 & $<0,0001$ & 9,88 \\
Rendimento de coxa+sobrecoxa & $\mathrm{Y}=-0,30 \mathrm{x}+20,93$ & 0,4634 & 0,0023 & 3,18 \\
\cline { 2 - 6 } & \multicolumn{1}{c}{42 dias } & 0,7314 & $<0,0001$ & 9,44 \\
Peso de carcaça & $\mathrm{Y}=-149 \mathrm{x}+2.228,33$ & 0,6233 & $<0,0001$ & 2,35 \\
Rendimento de carcaça & $\mathrm{Y}=-0,89 \mathrm{x}+77,49$ & 0,7693 & 0,0018 & 10,54 \\
Peso de peito & $\mathrm{Y}=-62,74 \mathrm{x}+797,17$ & 0,5272 & 0,0011 & 5,77 \\
Rendimento de peito & $\mathrm{Y}=-0,76 \mathrm{x}+27,65$ & 0,7572 & $<0,0001$ & 8,58 \\
Peso de coxa+sobrecoxa & $\mathrm{Y}=-43,48 \mathrm{x}+659,32$ & - & 0,3583 & 4,25 \\
Rendimento de coxa+sobrecoxa & $\mathrm{Y}=22,39$ & &
\end{tabular}

Aos 35 dias de idade, todas as variáveis estudadas foram influenciadas $(\mathrm{P} \leq 0,05)$ pela inclusão de pectina. PV, PC, PP, RP e PC+S apresentaram efeito quadrático em função da porcentagem de pectina, enquanto $\mathrm{RC}$ e $\mathrm{RC}+\mathrm{S}$ foram descritos por efeito linear decrescente (Tab. 6). Para as três variáveis de peso, os maiores valores foram obtidos com níveis abaixo de $1 \%$ de pectina, e os menores para $5 \%$, sendo estimados os níveis de $0,315,0,502$ e $0,578 \%$ de pectina para promover o valor máximo dessas variáveis. Para RP, os maiores valores ocorreram entre 1 e $3 \%$ de pectina, e os menores para $5 \%$. $\mathrm{O} \mathrm{RC}$ e o $\mathrm{RC}+\mathrm{S}$ diminuíram com o aumento dos níveis de pectina, sendo seus menores valores apresentados por aves que ingeriram ração contendo $5 \%$ de fibra.

Aos 42 dias de idade, apenas o $\mathrm{RC}+\mathrm{S}$ não foi influenciado significativamente $(\mathrm{P}>0,05)$ pela adição de pectina (Tab. 6). Todas as demais características estudadas diminuíram linearmente em função da porcentagem da fibra $(\mathrm{P} \leq 0,05)$, sendo seus menores valores observados com $5 \%$ de pectina.

\section{DISCUSSÃO}

Independentemente da porcentagem de pectina ingerida, o consumo de ração, o ganho de peso e a conversão alimentar semanais aumentaram até a idade de abate. Isso indica que nenhum dos níveis de fibra utilizados inibiu o crescimento dos frangos e que esses apresentaram piora na conversão alimentar com a idade. GonzálezAlvarado et al. (2010), ao avaliarem os efeitos contínuos da inclusão de casca de aveia e polpa de beterraba para frangos de corte, observaram pior desempenho das aves que ingeriram polpa de beterraba quando esta foi comparada à dietacontrole e à casca de aveia.

O nível de pectina na ração influenciou o desempenho dos frangos a partir da terceira semana de vida. Entretanto, os dados numéricos mostram piora na CA com o aumento do nível de pectina nas semanas seguintes. Essas alterações no $\mathrm{CR}$ e na $\mathrm{CA}$ respondem pelas diferenças de $150 \mathrm{~g}$ e de $500 \mathrm{~g}$ no GP observadas aos 35 e 42 dias, respectivamente, entre os frangos que não ingeriram $(0 \%)$ e os que ingeriam $5 \%$ de pectina. Como esperado, o efeito negativo da ingestão de $5 \%$ de pectina sobre o CR e a CA ocorreu acentuadamente a partir da quarta semana (28 dias de idade), período em que a linhagem utilizada apresentou seu maior GP. Redução no consumo diário de ração com a inclusão de carboidratos solúveis na ração também foi observada em aves por Razdan e Pettersson (1994) e Jiménez-Moreno et al. (2010), e em suínos por Anguita et al. (2007). Carboidratos solúveis, principalmente os ricos em pectina, 
retêm maior quantidade de água, gerando maior bolo alimentar (Bach Knudsen, 2001), o que promove dilatação no trato gastrintestinal (Serena et al., 2007) e aumento da viscosidade da digesta ( Silva, 2010), reduzindo o consumo de ração pelas aves. A piora na CA com o aumento no nível de pectina deve ser resultante da diminuição concomitante no $\mathrm{CR}$, bem como da piora na digestibilidade dos nutrientes com o aumento na adição da fibra na ração para a fase de crescimento dos frangos, conforme observado por Silva et al. (2009).

De acordo com os dados, nas duas idades analisadas, os maiores PV e GP total foram apresentados pelas aves que ingeriram $1 \%$ de pectina. Esses resultados indicam que níveis baixos de pectina $(\leq 1 \%)$ podem ser utilizados com o objetivo de melhorar o desempenho dos frangos, mas também apontam que níveis acima de $1 \%$ o influenciam negativamente. Os resultados deste estudo corroboram os de Gonzalez-Alvarado et al. (2007) e JimenezMoreno et al. (2009 e 2010), os quais verificaram melhora no ganho de peso nos frangos de corte com níveis baixos de diferentes fontes de fibra.

A VC não foi afetada pelos níveis de pectina utilizados no presente trabalho, indicando, dessa forma, que as porcentagens empregadas não promoveram deficiência nutricional severa, a ponto de levar os frangos a óbito antes da idade de abate.

A ingestão de água na terceira semana de idade não foi influenciada pela ingestão de pectina. Entretanto, na mesma semana, a relação CAg:CR aumentou com o aumento dos níveis da fibra, provavelmente em função da diminuição observada no CR. Na sexta semana, diferentemente do observado na terceira, a ingestão de água aumentou com os níveis de pectina. Isso deve ter ocorrido pelo fato de o CR dos frangos ter sido muito maior na sexta semana do que na terceira. Na sexta semana, a relação CAg:CR também foi maior com o aumento dos níveis de pectina. $\mathrm{O}$ aumento dessa variável deve ter sido provocado pelo aumento no CAg e concomitante diminuição no $\mathrm{CR}$ registrados no presente estudo em função do aumento do nível de pectina na ração. A relação CAg:CR estabelecida no Nutrient..., (1994) para frangos corresponde a $2,5 \mathrm{~mL}$ de água por grama de ração
ingerida.Sendo assim, os dados mostram que, na fase inicial, as aves que receberam $5 \%$ ingeriram volume de água por grama de ração maior que o estabelecido, o que não foi observado para as demais aves cujo consumo hídrico ficou próximo ao valor de referência.

$\mathrm{Na}$ fase de crescimento, todas as aves que receberam pectina tiveram ingestão maior de água por grama de ração que a estabelecida para frangos, exceção feita às aves-controle. Polissacarídeos solúveis absorvem água e sofrem geleificação, sendo a viscosidade da solução gelatinosa dependente de fatores como: tamanho da molécula, ramificação ou não das moléculas, presença de cargas livres, das estruturas adjacentes e, claro, da concentração (Smits e Annison, 1996). Em baixas concentrações, os polissacarídeos aumentam a viscosidade pela interação direta com as moléculas da água. Em altas concentrações, os polissacarídeos interagem entre si formando uma rede (gel) (Morris e RossMurphy, 1981). O aumento na ingestão de pectina causa aumento na viscosidade intestinal dos frangos tanto na fase inicial como na de crescimento (Silva, 2010). Dessa forma, a maior ingestão de água por grama de ração apresentada pelos frangos na fase de crescimento em função do aumento dos níveis de pectina (presente estudo) deve ter sido provocada pelo aumento na viscosidade intestinal das aves.

O aumento na adição de pectina na ração diminuiu os pesos e os rendimentos de carcaça e de cortes dos frangos aos 35 e 42 dias. De acordo com Philip et al. (1995) e Classen (1996), a geleificação da fibra solúvel em contato com a água reduz o tempo de trânsito do alimento e funciona como barreira à ação hidrolítica das enzimas, o que dificulta o contato dessas com as moléculas de amido, proteicas e lipídicas do alimento e diminui o contato do bolo alimentar com as células absortivas da membrana intestinal. Segundo Silva (2010), o aumento dos níveis de pectina na ração resulta em aumento na viscosidade intestinal dos frangos e redução no tempo de trânsito intestinal na fase de crescimento. Esses efeitos provocam redução na digestão e na absorção dos nutrientes da ração, o que deve ter piorado a conversão alimentar e, consequentemente, causado a redução no crescimento, no peso e no rendimento de carcaça e de cortes das aves alimentadas com o mais alto nível de pectina na ração observada neste 
experimento. O aumento da viscosidade da digesta e da excreção de fezes pegajosas causado pela adição de polissacarídeos não amiláceos (PNA) na ração dos monogástricos (Iji, 1999) é considerado o principal efeito dos PNA sobre a produtividade (Classen e Bedford, 1991; Smits e Annison, 1996), uma vez que estes podem levar a alterações na estrutura e função da mucosa intestinal (Johnson e Gee, 1986; Brunsgaard e Eggum, 1995).

\section{CONCLUSÃO}

A ingestão contínua de até $1 \%$ de pectina na ração mantém o desempenho máximo das aves. O consumo contínuo acima desse nível de fibra prejudica os parâmetros zootécnicos e aumenta o consumo de água na fase de maior crescimento.

\section{AGRADECIMENTOS}

Os autores agradecem à Fundação de Amparo à Pesquisa (FAPESP), pelo auxílio financeiro ao projeto de pesquisa (Proc. $\mathrm{N}^{\circ}$ 07/59891-0), e à Coordenação de Aperfeiçoamento de Pessoal de Nível Superior (CAPES), pela concessão da bolsa de estudos.

\section{REFERÊNCIAS}

ANGUITA, M.; GASA, J.; NOFRARIAS, M. et al. Effect of coarse ground corn, sugar beet pulp and wheat bran on the voluntary intake and physicochemical characteristics of digesta of growing pigs. Livest. Sci., v.107, p.182-191, 2007.

BACH KNUDSEN, K.E. The nutritional significance of "dietary fibre" analysis. Anim. Feed Sci. Technol., v.90, p.3-20, 2001.

BOARO, M. Morfofisiologia do trato intestinal. In: CONFERÊNCIA APINCO DE CIÊNCIA E TECNOLOGIA AVÍCOLA, 2009, Porto Alegre, RS. Anais... Porto Alegre: FACTA. 2009, p.261-274.

BRUNSGAARD, G.; EGGUM, B.O. Caecal and colonic tissue structure and proliferation as influenced by adaptation period and indigestible polysaccharides. Comp. Biochem. Physiol., v.112, p.573-583, 1995.

CARR, T.P.; WOOD, K.J.; HASSEL, G.A. et al. Raising intestinal contents viscosity leads to greater excretion of neutral steroids but not bile acids in hamsters and rats. Nutr. Res., v.23, p.91-102, 2003.

CLASSEN, H.L. Cereal grain starch and exogenous enzymes in poultry diets. Anim. Feed Sci. Technol., v.62, p.21-27, 1996.
CLASSEN, H.L.; BEDFORD, M.R. The use of enzymes to improve the nutritive value of poultry feeds. In: HARESIGN, W.; COLE, D.J.A. (Ed.). Recent Advances in Animal Nutrition. Nottingham: UK, 1991. p.95-116.

DELZENNE, N.M.; DAUBIOUL, C.; NEYRINCK, A. et al. Inulin and oligofructose modulate lipid metabolism in animals: review of biochemical events and future prospects. Br. J. Nutr. v.2, p.255-259, 2002.

FIETZ, V.R.; SALGADO, M.S. Efeito da pectina e da celulose nos níveis séricos de colesterol e triglicerídeos em ratos hiperlipidêmicos. Cienc. Tecnol. Aliment., v.19, p.3218-3221, 1999.

GONZÁLEZ-ALVARADO, J.M.; JIMÉNEZMORENO, E.; GONZÁLEZ-SÁNCHEZ, D. et al. Effect of inclusion of oat hulls and sugar beet pulp in the diet on productive performance and digestive traits of broilers from 1 to 42 days of age. Anim. Feed Sci. Technol., v.162, p.37-43, 2010.

GONZALEZ-ALVARADO, J.M.; JIMENEZMORENO, E.; LAZARO, R.; MATEOS, G.G. Effect of type of cereal, heat processing of the cereal and inclusion of fiber in the diet on productive performance and digestive traits of broilers. Poult. Sci., v.86, p.1705-1715, 2007.

IJI, P.A. The impact of cereal non-starch polysaccharides on intestinal development and function in broiler chickens. Worlds Poult. Sci. J., v.55, p.375-388, 1999.

JIMÉNEZ-MORENO, E.; $\quad$ GONZÁLEZALVARADO, J.M.; LÁZARO, R.; MATEOS, G.G. Effects of type of cereal, heat processing of the cereal, and fiber inclusion in the diet on gizzard $\mathrm{pH}$ and nutrient utilization in broilers at different ages. Poult. Sci., v.88, p.1925-1933, 2009.

JIMENEZ-MORENO, E.; GONZALEZALVARADO, J.M.; GONZALEZ-SANCHEZ, D. et al. Effects of type and particle size of dietary fiber on growth performance and digestive traits of broilers from 1 to 21 days f age. Poult. Sci., v.89, p.2197-2212, 2010.

JOHNSON, I.T.; GEE, J.M. Gastrointestinal adaptation in response to soluble non-available polysaccharides in the rat. Br. J. Nutr., v.55, p.497505, 1986.

JUŚKIEWICZ, J.; JANKOWSKI, J.; ZDUŃCZYK, Z.; MIKULSKI, D. Performance and gastrointestinal tract metabolism of turkeys Fed diets with different contents of fructooligosaccharides. Poult. Sci., v.85, p.886-891, 2006. 
MORRIS, E.R.; ROSS-MURPHY, S.B. Chain flexibility of polysaccharides and glycoproteins from viscosity measurements. In: NORTHCOTE, D.H. (Ed). Techniques in Carbohydrate Metabolism. Elsevier: Amsterdam, 1981. p.1-46.

NUTRIENT requirements of poultry. NRC. 9.ed. Washington: National Academy of Sciences, 1994. $157 \mathrm{p}$.

PHILIP, J.S.; GILBERT, H.J.; SMITHARD, R.R. Growth, viscosity and beta-glucanase activity of intestinal fluid in broiler chickens feed on barleybased diets with or without exogenous beta-glucanase. Br. Poult. Sci., v.36, p.599-605, 1995.

RAZDAN, A.; PETTERSSON, D. Effect of chitin and chitosan on nutrient digestibility and plasma lipid concentrations in broiler chickens. Br. J. Nutr., v.72, p.277-288, 1994.

ROSA, A.P.; UTTPATEL, R. Uso de enzimas nas dietas para frangos. In: SIMPÓSIO BRASIL SUL DE AVICUlTURA, 8., 2007, Chapecó, SC. Anais... Chapecó: Embrapa Suínos e Aves. 2007. p.102-115.

ROSTAGNO, H.S., ALBINO, L.F.T., DONZELE, J.L. et al. Tabelas brasileiras para aves e suínos: composição de alimentos e exigências nutricionais. 2.ed. Viçosa: Universidade Federal de Viçosa, 2005. 186p.

SAKI, A.A.; MATIN, H.R.H.; TABATABAI, M.M. et al. Microflora population, intestinal condition and performance of broilers in response to various rates of pectin and cellulose in the diet. Arch. Geflugelk., v.74, p.183-188, 2010.

SATOH, H.; HARA, T.; MURAKAWA, D. et al. Soluble dietary fiber protects against nonsteroidal antiinflammatory drug-induced damage to the small intestine in cats. Dig. Dis. Sci., v.55, p.1264-1271, 2010.

SERENA, A.; HEDEMANN, M.S.; BACH KNUDSEN, K.E. Feeding high fiber diets changes luminal environment and morphology in the intestine of sows. Livest. Sci., v.109, p.115-117, 2007.
SILVA, V.K. Pectina na ração de frangos de corte: digestibilidade, parâmetros zootécnicos e metabolismo lipídico. 2010. 140f. Tese (Doutorado em Zootecnia) Faculdade de Ciências Agrárias e Veterinárias, Universidade Estadual Paulista, Jaboticabal.

SILVA, V.K.; MORITA, V.S.; BOLELI. I.C. Digestibilidade de rações para frangos de corte contendo pectina. In: CONFERÊNCIA APINCO DE CIÊNCIA E TECNOLOGIA AVÍCOLA, 2009, Porto Alegre, RS. Anais... Porto Alegre:FACTA. Cd-Rom (Resumo).

SMITS, C.H.M.; ANNISON, G. Non-starch polysaccharides in broiler nutrition - towards a physiologically valid approach to their determination. World's Poult. Sci. J. v.52, p.203-221, 1996.

SMITS, C.H.M.; VELDMAN, A.; VERSTEGEN, M.W.A et al. Dietary Carboxymethyl cellulose with high instead of low viscosity reduces macro nutrient digestion in broiler chickens. J. Nutrit., v.127, p.483487, 1997

STATISTICAL Analysis System: user's guide. SAS INSTITUTE. Version 9.1 ed. Cary, 2002.

TAKAHASHI, T.; SAKATA, T. Crystalline cellulose reduces plasma glucose concentrations and stimulates water absorption by increasing the digesta viscosity in rats. J. Nutr., v.135, p.2405-2410, 2005.

TEIRLYNCK, E.; BJERRUM, L.; EECKHAUT, V. et al. The cereal type in feed influences gut wall morphology and intestinal immune cell infiltration in broiler chickens. Br. J. Nutr., v.102, p.1453-1461, 2009

VAN DER KLIS, J.D.; VERSTEGEN, M.W.; VAN VOORST, A. Effect of a soluble polysaccharide (carboxy methyl cellulose) on the absorption of minerals from the gastrointestinal tract of broilers. $\mathrm{Br}$. Poult. Sci, v. 34, p. 985-997, 1993. 